\title{
Anomalous Diffusion with Step-Function Injection in a Radial Geometry
}

\author{
S. Wang ${ }^{1,2, \dagger}$ M. Zhao ${ }^{2,1}$ and X. $\mathrm{Li}^{3}$ \\ ${ }^{1}$ Department of Geological Science, University of Alabama, 35487 Tuscaloosa, USA \\ ${ }^{2}$ Geotechnical and structural engineering research center, Shandong University, Jinan 250061, P.R. China \\ ${ }^{3}$ Department of Engineering Mechanics, Shandong University, Jinan 250061, P.R. China \\ ${ }^{4}$ School of Mathematical Sciences, University of Jinan, 250100 Jinan, P.R. China
}

†Corresponding Author Email: shaoweiwang@sdu.edu.cn

(Received October 31, 2013; accepted June 10, 2014)

\begin{abstract}
A generalized advection dispersion model with time-fractional derivative is developed for analyzing underground injection of wastes. The governing equations for a cylindrically symmetrical system are cast in nondimensional form and then transformed and solved in Laplace space. With the help of Crump algorithm, the Laplace space solution is inverted. The effect of fractional derivative parameter to the particle movement process is discussed numerically, and it is pointed out that the role of fractional derivative parameter is to slow the particle motion when $0<\beta<1$.
\end{abstract}

Keywords: Radial diffusion, Fractional derivative, Laplace transform, Advection dispersion equation, Crump algorithm.

\section{NOMENCLATURE}

$\begin{array}{ll}A & \text { uniform throughout medium } \\ C & \begin{array}{l}\text { tracer concentration } \\ \text { radial dispersion coefficient }\end{array} \\ D_{r} & \\ \mathrm{R} & \text { retardation factor } \\ Q & \text { volumetric injection rate }\end{array}$

\section{INTRODUCTION}

Many researchers have pointed out that the tracer particles at the Earth surface spend more time at rest than in motion (Sadler 1981; Tipper 1983), and many particles do not move from the point of tracer input over the measurement period because of the frequency distributions of bed load path length in the porous media (Pryce and Ashmore 2003; Abdullah et. al. 2008). The heavy-tailed waiting times will affect longterm dispersion rates, which results to the "anomalous" transport characteristics. Anomalous diffusion is frequently observed in disordered media. It is characterized by non-linear time dependence of the mean squared displacement

$$
r^{2}(t) \sim r^{\gamma},
$$

with $\gamma \neq 1$. For the case with $0<\gamma<1$, the process is called subdiffusive and such an anomaly is found in many physical systems including glasses, amorphous semiconductors, and porous media (Blumen and

$\begin{array}{ll}\boldsymbol{B} & \text { thickness } \\ R & \text { radial distance } \\ t & \text { time } \\ v & \text { radial seepage velocity } \\ A & \text { dispersivity } \\ \beta, \gamma & \text { fractional parameters }\end{array}$

Zumofen 1986; Scher and Montroll 1975; Wong 2004; Even and Jortner 1984).

To characterize the historical waiting time of particles movement, a continuous time random walk (CTRW), which imposes a random waiting time before the particle jump occurs, is introduced by Scher and Lax (1973). Based on the theory of CTRW, time-fractional advection dispersion equation is obtained (R. Schumer and Baeumer 2009)

$$
\frac{\partial^{\gamma} C(x, t)}{\partial t^{\gamma}}=-v \frac{\partial C(x, t)}{\partial x}+D \frac{\partial^{\alpha} C(x, t)}{\partial t^{\alpha}}
$$

In this work we present a generalized advection dispersion equation (ADE) with time fractional derivative, the proposed fractional $\mathrm{ADE}$ is solved to obtain analytical expressions in Laplace domain. Then the Laplace space solution is inverted with the help of Crump algorithm, which uses the real and imaginary parts of a Fourier series. 


\section{Mathematical Model}

The model presented here assumes that the contaminants are injected in a step-function manner for a finite period and transported by means of advection and dispersion while undergoing linear sorption and retardation. On the basis of principle of conservation of mass and the above assumptions, the time-fractional governing equation (in radial coordinates) for transport for an injected contaminant can be written as:

$R \tau_{0} \frac{\partial^{\beta} C}{\partial t^{\beta}}=\frac{1}{r} \frac{\partial}{\partial r}\left(r D_{r} \frac{\partial C}{\partial r}\right)-v \frac{\partial C}{\partial r}-\lambda R C$

Here, $C(r, t)$ is the tracer concentration $\left[M L^{-3}\right]$ in the fluid phases at a radial distance $r[L]$ and time $t[T]$, $D_{r}$ is radial dispersion coefficient $\left[L^{2} T^{-1}\right], v$ is the radial seepage velocity $\left[L T^{-1}\right]$, and $\lambda$ is first-order decay constant $\left[T^{-1}\right], \quad R$ is the contaminant's retardation factor and is used to account for the sorption mechanism in solute transport, which is a constant. In addition, in order to balance the dimension in both sides of Eq.(3), $\tau_{0}$ is introduced as a fractional characteristic time $\left[T^{\beta-1}\right]$ because of the definition of fractional calculus,

$$
\frac{\partial^{\beta}}{\partial t^{\beta}} f t=\frac{1}{\Gamma(\beta)} \int_{0}^{t} \frac{f^{\prime}(\tau)}{(t-\tau)^{1-\beta}} d \tau, 0<\beta<1
$$

For radial injection with a constant fluid flux, the seepage velocity $\mathrm{V}$ at any point $r$ is obtained from the continuity equation (Tang and Babu 1979), which can be given by the expression

$v=\frac{A}{r}=\frac{Q}{2 \pi b \phi r}$

where $Q$ is the volumetric injection rate and $b$ is the thickness of the receiving formation, $\phi$ is the effective porosity of the porous medium and $A$ is uniform throughout medium

$$
A=\frac{Q}{2 \pi b \phi}
$$

Dispersion is assumed to be a function the seepage velocity and dispersivity $\alpha$

$$
D_{r}=\alpha v=\frac{\alpha A}{r}
$$

The initial and boundary conditions are

$$
\begin{aligned}
& C r, 0=0 \\
& C 0, t=C_{0} H(t-\Delta t) \\
& C(r, t) \rightarrow 0, r \rightarrow \infty
\end{aligned}
$$

where $H(t-\Delta t)$ is the Unit step function, $\Delta t$ is an arbitrary time. The Unit step function has a sharp front (i.e., a first derivative discontinuity) at $t=\Delta t$.

\section{Solution Of The Model}

Introducing the following dimensionless variables

$$
r^{*}=\frac{r}{\alpha}, \quad r^{*}=\frac{r}{\alpha}, \quad \tau=\frac{A t}{R \alpha^{2}} .
$$

For simplicity of notation, the asterisk will be omitted hereinafter. With these substitutions, equation (3) becomes

$\eta \frac{\partial^{\beta} C}{\partial t^{\beta}}=\frac{1}{r} \frac{\partial^{2} C}{\partial r^{2}}-\frac{1}{r} \frac{\partial C}{\partial r}-\frac{\lambda \alpha^{2} R}{A} C$

where $\eta=\tau_{0}\left[\frac{A}{R \alpha^{2}}\right]^{1-\beta} \quad$ is a dimensionless constant.

Equation (12) can be solved by using the method of Laplace transforms, producing the following result

$$
\begin{aligned}
& \frac{1}{r} \frac{d^{2} \bar{C}}{d r^{2}}-\frac{1}{r} \frac{d \bar{C}}{d r}-\left(\eta s^{\beta}+\frac{\lambda \alpha^{2} R}{A}\right) \bar{C}=0 \\
& \bar{C} 0, s=C_{0} e^{-\Delta t s} / s \\
& \bar{C}(r, s) \rightarrow 0, r \rightarrow \infty
\end{aligned}
$$

where

$$
\bar{C}=\int_{0}^{\infty} \exp (-s t) C(r, t) d t=\bar{C}(r, s)
$$

If we introduce the new variables

$$
\bar{C}=e^{r / 2} y(\xi) \text { and } \xi=P^{-2 / 3}\left(r P+\frac{1}{4}\right)
$$

where $P=\eta s^{\beta}+\frac{\lambda \alpha^{2} R}{A}$, then the Eq.(13) can be rewritten as

$$
\frac{d^{2} y}{d \xi^{2}}-\xi y=0
$$

and its solutions is

$$
y \xi=C_{1} A i \xi+C_{2} B i(\xi)
$$

where $A i(\xi)$ and $B i(\xi)$ are the Airy functions of the first and second kind, respectively, both are Bessel functions of the one-third order, and $C_{1}$ and $C_{2}$ are constants. So, the solution to Equation (13) in Laplace domain is

$$
\begin{array}{r}
\bar{C} r, s=C_{1} e^{r / 2} A i\left(\left[r+\frac{1}{4 P}\right] P^{1 / 3}\right)+ \\
C_{2} e^{r / 2}(\xi) B i\left(\left[r+\frac{1}{4 P}\right] P^{1 / 3}\right)
\end{array}
$$

Constants $C_{1}$ and $C_{2}$ in Equation (20) can be determined with the help of initial and boundary conditions (8) - (10) of the problem, which yields 


$$
\begin{aligned}
& \bar{C} r, s=e^{r / 2}\left(\frac{1-e^{-\Delta t s}}{s}\right) \frac{1}{A i\left(\frac{1}{4}\left(\eta s^{\beta}+\frac{\lambda \alpha^{2} \mathrm{R}}{\mathrm{A}}\right)^{-2 / 3}\right.} \times \\
& A i\left(\frac{1}{4}\left(\eta s^{\beta}+\frac{\lambda \alpha^{2} \mathrm{R}}{\mathrm{A}}\right)^{-2 / 3}+r\left(\eta s^{\beta}+\frac{\lambda \alpha^{2} \mathrm{R}}{\mathrm{A}}\right)^{1 / 3}\right)
\end{aligned}
$$

\section{Numerical RESUlts AND Discussion}

Because of the difficulty to get the analytical inversion of Laplace space equations back to real time and space, a number of numerical methods have been developed to calculate these inverses. Of these methods, the Stehfest algorithm is one of the best known. However, for the present model, this method will produce spurious results because of the sharp amplitude discontinuity of the step-function source term. On the other hand, the choice of Laplace-inversion algorithm is made on the basis of computational efficiency.

It has been pointed out that much better computational accuracy can be obtained by using the real portion of a Fourier series to invert the Laplace space solution. Here the solution is inverted to the time domain numerically using a scheme developed by Crump (1976), which is presented for numerically inverting a Laplace transform that requires only sine, cosine, and exponential functions. The Crump algorithm offers a significant advantage over other numerical methods in that the number of $s$ values for which the Laplace-transformed equations must be solved is independent of the number of time stations at which the solution is required. The Crump inversion can be written as

$$
\begin{aligned}
f(t) \approx & \frac{e^{c t}}{2 T_{m}}\left(\frac{F c}{2}+\sum_{k=1}^{\infty}[\operatorname{Re} F c+d i \cos d t\right. \\
& -\operatorname{Im} F c+d i \sin d t]
\end{aligned}
$$

where $f(t)$ is the function in real time space, $i$ is imaginary unit, $T_{m}$ is the maximum time of the calculation, $F(c)$ is the Laplace space solution as a function of $c, R e$ and $I m$ is the real and imaginary part of a function, respectively.

Based on the Crump algorithm, a Mathematica program was written to evaluate the concentration distribution with respect to time (or distance). We assume that a step-function injection of contaminant is transported in the fracture system with a dimensionless duration of 250, i.e., $\Delta t=250$, which is shown in Fig.1. It shows that the numerical result is excellent for the abrupt change of the step-function source.

Fig.2 and Fig.3 present concentration distribution profile, respectively, at $r / \alpha=5$ and $r / \alpha=15$ for different $\beta$. As an additional illustration of the effect of fractional derivative parameter $\beta$, we will consider the case what is the concentration value with respect to

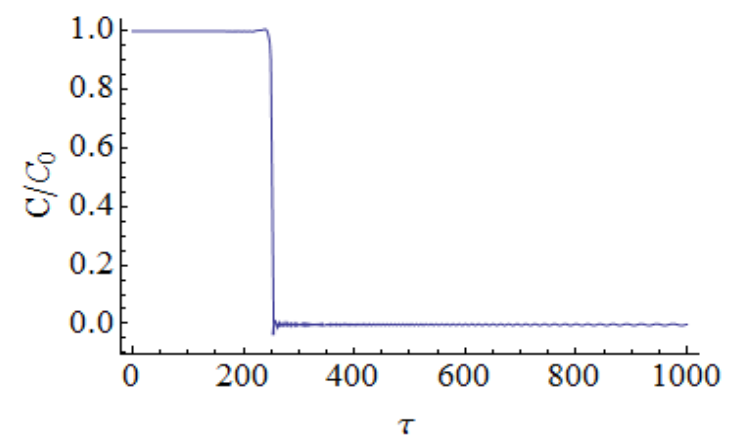

Fig. 1. Concentration as a function of dimensionless time $(\tau)$ at $r / \alpha=0$ for a step-function source that has a duration $\Delta \tau=\mathbf{2 5 0}$.

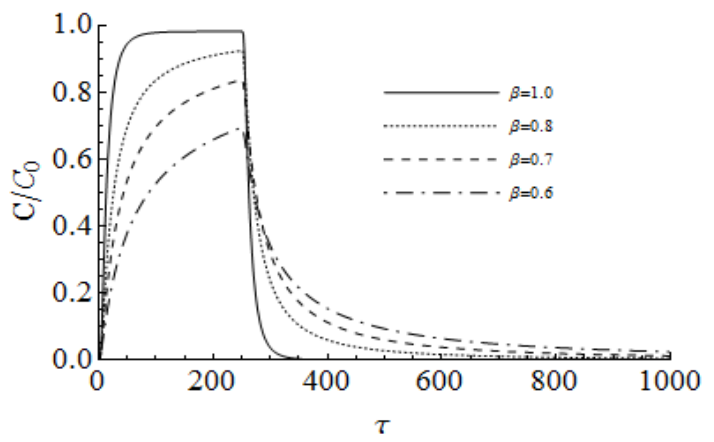

Fig. 2. Effect of $\beta$ on the distribution of concentration with respect to time $\tau$ at $r / \alpha=5$ when $\lambda=\mathbf{0 . 1}$.

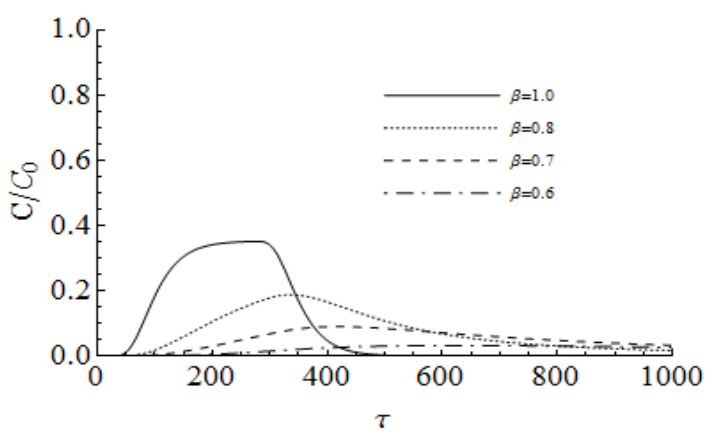

Fig. 3. Effect of $\beta$ on the distribution of concentration with respect to time $\tau$ at $r / \alpha=15$ when $\lambda=0.1$.

time at different distance for given $\beta$. This situation is illustrated in Fig.4. In contrast to the classical advection dispersion model, the solute movement is slower for the fractional ADE mode, which means less particles arrive the given distance in the same duration for a fractional ADE model. And the concentration value at given distance decreases with the decreasing fractional derivative parameter $\beta$.

To further illustrate the role of fractional derivative, consider Fig.5, which shows the concentration distribution as a function of dimensionless distance $r / a$ for different $b$ at given dimensionless time. Inspection of Fig. 5 reveals a distinct trend of decreasing arrival distance with the decreasing fractional derivative parameter b. This result is consistent with Eq.(1). 

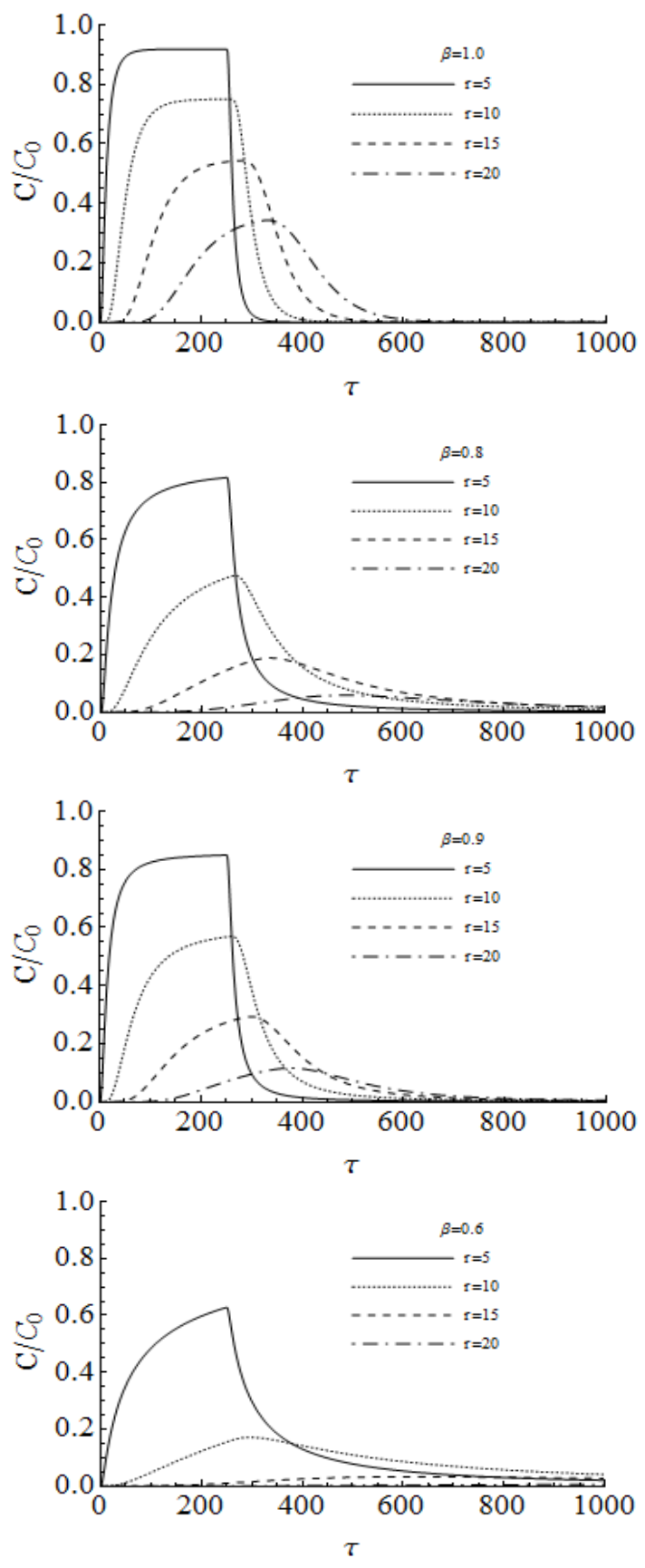

Fig. 4. Concentration at different dimensionless distances as a function of dimensionless time $\tau$ for different $\beta$.

\section{CONClusion}

The present paper presented an analytical solution to the generalized radial advection dispersion equation with time-fractional derivative for a step-function source injection. The governing equation was made nondimensional and solved in Laplace space. Then the results were inverted numerically using Crump algorithm, and the simulations of numerical results are given with the help of developed Mathematica program. The effect of fractional derivative parameter to the particle movement was discussed, and we found that the
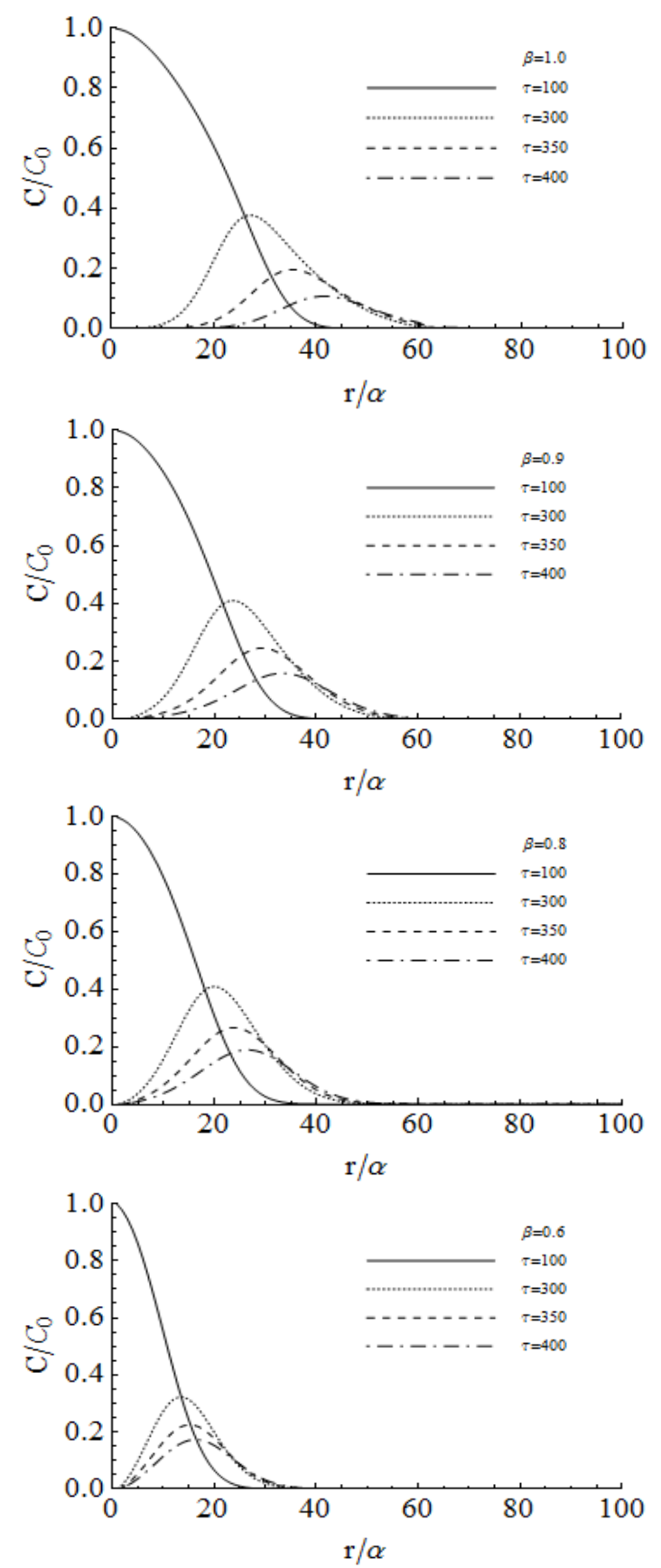

Fig. 5. Concentration at different dimensionless time as a function of dimensionless distance $r / \alpha$ for different $\beta$.

decreasing fractional derivative parameter slow the solute motion.

\section{ACKNOWLEDGEMENTS}

The authors appreciate very much the constructive comments given by the anonymous reviewers. This work is supported by the National Natural Science Foundation of China (Nos.11002083, 11002049 and 91130017), and the National Basic Research Program of China (2013CB036000). 


\section{REFERENCES}

Abdullah, S., W.H. Kurniawan, and A. Shamsudeen (2008). Numerical analysis of the combustion process in a compressed natural gas direct injection engine. Journal of Applied Fluid Mechanics, 1(2), 65-86.

Blumen, A., J. Klafter, and G. Zumofen (1986). Models for reaction dynamics in glasses. In I. Zschokke (Ed.), In Optical Spectroscopy of Glasses, Dordrecht, Holland, pp. 199-265. Springer Netherlands.

Crump, K. (1976). Numerical inversion of Laplace transforms using a Fourier series approximation. $J$. ACM 23(1), 89-96.

Even, U., K. Rademann, J. Jortner, et al., (1984). Electronic energy transfer on fractals. Phys. Rev. Lett. 52, 2164-2167.

Pryce, R. and P. Ashmore (2003). The relation between particle path length distributions and and channel morphology in gravel-bed streams: A synthesis. Geomorphology 56, 167-187.

Sadler, P. (1981). Sediment accumulation rates and the completeness of stratigraphic sections. J. Geol. 89, 569-584.
Scher, H. and M. Lax (1973). Stochastic transport in a disordered solid. I. Theory. Phys. Rev. B 7(10), 4491-4502.

Scher, H. and E. Montroll (1975). Anomalous transit time dispersion in amorphous solids. Phys. Rev. B $12,2455-2477$.

Schumer, R., M.M. Meerschaert, and B. Baeumer (2009). Fractional advection-dispersion equations for modeling transport at the earth surface. $J$. Geophys. Res. 114, F00A07.

Tang, D. and D. Babu (1979). Analytical solutions of a velocity dependent dispersion problem. Water Resorces Res. 15(6), 1471-1478.

Tipper, J. (1983). Sediment accumulation rates and the completeness of stratigraphic sections. Nature 302(5910), 696-698.

Wong, I.Y., M.L. Gardel, D.R. Reichman, et al., (2004). Anomalous diffusion probes microstructure dynamics of entangled F-Actin networks. Phys. Rev. Lett. 92, 178101. 